\title{
Immunohistochemical examination of p97/VCP expression in developing mouse pancreas and liver
}

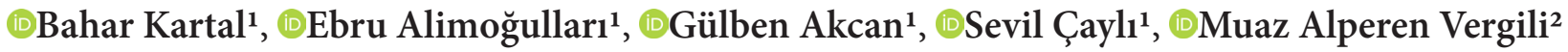 \\ ${ }^{1}$ Ankara Yıldırım Beyazıt University, Faculty of Medicine, Department of Histology and Embryology, Ankara,Turkey \\ ${ }^{2}$ Ankara Yildırım Beyazıt University, Faculty of Medicine, Ankara,Turkey
}

Cite this article as: Kartal B, Alimoğulları E, Akcan G, Çaylı S, Vergili MA. Immunohistochemical examination of p97/VCP expression in developing mouse pancreas and liver. J Health Sci Med 2021; 4(3): 334-339.

\begin{abstract}
Aim: The 97-kDa Valosin-containing protein has important functions in proteolysis. Although the expression of p97/VCP has been studied in many types of cells and tissues, the localization of p97/VCP at cellular level in developing mouse pancreas and liver has not been determined. Therefore, the aim of our study was investigate the immuolocalization of p97/VCP in the mouse fetal and postnatal liver and pancreas.

Material and Method: The liver and pancreas from fetal, postnatal (1, 5, 15, 35 days old ) and adult (50 days old) mice were examined by using immunohistochemistry in order to determine the expression of p97/VCP. Furthermore the development of mouse pancreas and liver were histomorphologically analyzed under light microscope.

Results: The histological structures of the mouse pancreas and liver were maintained in postnatal period. The histological differences between endocrine and exocrine pancreas were significantly observed from the postnatal 5th day. The expression of p97/VCP in Langerhans islets was determined from day 5. p97/VCP expression was also seen in the exocrine pancreas in all postnatal days. $\mathrm{p} 97 / \mathrm{VCP}$ was expressed in developing pancreatic and liver tissues and its expression was increased with the development.

Conclusion: This study is the first to examine the distribution and the localization of p97/VCP in the fetal and postnatal mouse liver and pancreas. This study forms the basis for clinical studies in order to determine the function of p97/VCP in liver and pancreatic cells.
\end{abstract}

Keywords: p97/VCP, postnatal development, immunohistochemistry pancreas, liver

\section{INTRODUCTION}

Protein degradation is a mechanism which allows proteins in the cell to function at their normal levels called steady-state. Protein degradation occurs both in cytoplasm and nucleus by ubiquitin proteasome system (UPS) and its regulatory proteins in eukaryotes. These regulatory proteins includes COP9 signalosome $(\mathrm{CSN})$ and p97/Valosin containing protein (VCP) which control the destruction of certain substrates (1). UPS has two basic phases, ubiquitination and the degradation of ubiquitinated proteins (2). UPS involves in the maintenance of cellular homeostasis, protein quality control, and the removal of misfolded or damaged proteins (3). In the ATP-dependent ubiquitin pathway, binding of ubiquitin to the target protein, this process called as ubiquitination which is carried out by (Ubiquitinactivating enzymes) E1, (ubiquitin-conjugating enzymes) E2 and (ubiquitin ligase) E3 (4). The aim of ubiquitination is to deliver the ubiquitinated proteins to lysosome, an autophagosomal vacuole, or a $26 \mathrm{~S}$ proteasome. The p97/VCP plays important roles in proteolysis due to the UPS. VCP serves as a chaperone protein in UPS. VCP is amember of , type II AAA (ATPases Associated with a variety of Activities) ATPase family $(5,6)$. VCP consists of four domains, the N-terminal domain $(\mathrm{N})$, two ATPase domains (D1 and D2) and the C-terminal domain $(\mathrm{C})$. While the $\mathrm{N}$-terminal domain is responsible for binding to the polyubiquitin chains and the substrate recognition, the D1 and D2 domains are responsible for the chaperone activity of the VCP (7-9). Ubiquitinated proteins can either be transferred directly to the proteasome or indirectly transferred by VCP. VCP and its cofactors, binds to the ubiquitized proteins and guides them by its chaperone activity before they are degraded in the $26 \mathrm{~S}$ proteasome. After the substrate is ubiquitized 
,VCP uses ATP to separate the protein complexes and directed to the proteasome.p97/VCP has associated with a variety of cellular protein pathways, including nuclear envelope reconstruction, cell cycle regulation, Golgi reassembly, suppression of apoptosis, DNA damage responses, maturation of autophagosome and sperm capacitation $(10,11)$. In addition, during endoplasmic reticulum-associated degradation, p97/VCP dislodges ubiquitinated proteins from the endoplasmic reticulum (ER) and chaperones them to the cytosol for proteasomal degradation (12). For ubiquitination of misfolded proteins in the ER, interaction with p97/VCP is required $(13,14)$.

Although p97/VCP has been studied in different tissues, its presence and distribution during postnatal development of mouse pancreas and liver remains to be elucidated. Therefore the aim of the present study was to investigate the cellular localization p97/VCP in the fetal, postnatal and adult mouse liver and pancreas.

\section{MATERIAL AND METHOD}

\section{Animals and Experimental Design}

We used twenty-four male Balb C mice at fetal, postnatal ages of $1,5,15,35$ and adult 50 days (six mice per group), i.e., corresponding to infant (5), prepubertal (15 day), pubertal (30 day), and adult (50 days) periods were obtained from Kobay Animal Research Laboratory. This study was approved by Kobay Animal Research Laboratory Local Ethics Committee (Date: 23.03.18, Decision No: 277). The mice were cared in the laboratory according to institutional guidelines and the Guide for Care and Use of Laboratory Animals of the National Research Council. All mice were maintained in a temperature-controlled room $\left(20-23^{\circ} \mathrm{C}\right)$ on a $12 \mathrm{~h}$ light/ dark cycle with food and fresh water available adlibitum. Animals were sacrificed using anaesthesia with ketamine hydrochloride (40 mg/kg) (Ketalar, Eczacibasi, Istanbul, Turkey) and xylazine hydrochloride (5 mg/kg) (Rompun, Bayer, Istanbul, Turkey), and liver and pancreatic tissues were removed.

\section{Histological Procedure}

Liver and pancreatic tissues of fetal, postnatal $(1,5,15$, 35 days) and adult (50 days) mice were fixed in 10\% formalin and after routine histological procedures, the samples were embedded in paraffin. $5 \mu \mathrm{m}$ thick sections were obtained from each paraffin blocks and stained with haematoxylin-eosin (H\&E). The slides were histologically evaluated under light microscope (LeicaDM4000, Wetzlar, Germany).

\section{Immunohistochemical Analysis}

Slides were deparaffinized, put in xylene and rehydrated in a graded series of ethanol. Antigen retrieval was performed in a microwave oven with citrate buffer and the tissues were then blocked in blocking serum (Ultra V Block, ScyTek Laboratories, Utah, USA). The slides were incubated with the mouse monoclonal p97/VCP (ab11433, 1: 500, Abcam, UK) primary antibody, $1 \mathrm{~h}$ at room temperature. After incubation with primary antibody, the tissue sections were washed twice with PBS for 5 minutes each time, and then, incubated with biotinylated anti-mouse (BA-9200; 1:400 Dilution; Vector Laboratories, Burlingame, CA) seconder antibody for 10 minutes at room temperature. After three washes with PBS, the antigen- antibody complexes were detected using a streptavidin- peroxidase complex (TP-060-HL; LabVision, Fremont, CA, USA) for 10 minutes. Bound peroxidase was developed with 3-amino-9-ethyl-carbazol (AEC) (ScyTek Laboratories, USA) chromogen.Sections were counterstained with Mayer's hematoxylin (ScyTek Laboratories, Utah, USA) and mounted with Permount (Fisher Chemicals, Springfield, NJ, USA) on glass slides. Photographs were taken with a Leica microscope(Leica $\mathrm{DM}^{2} 500$, Nussloch, Germany). H-SCORE analyses were used for the immunohistochemistry evaluation as previously described (15).

\section{Statistical Analyses}

Twenty-four male Balb C mice were used in this study. Statistical analysis was performed by one-way ANOVA followed by Dunnett's test and the Mann Whitney U test by using Sigma Plot 12 (Jandel Scientific Corp., San Rafael, CA). The statistical significance of the data was defined as $\mathrm{p}<0.05$. All data are presented as the mean \pm standard error (SEM) of 3 independent experiments.

\section{RESULTS}

\section{Histological Results}

Histomorphological examinations showed that normal histologic structure of mouse liver maintained in postnatal period additionally liver lobulation was increased on day $15^{\text {th }}$. (Figure $1, \mathbf{G}, \mathbf{H}$ ). The presence of portal areas along with central vein provided evidence of a lobular structure of the mouse liver. Hepatocytes were arranged in cords, and radiated from the regions of central vein and extending to the portal areas. The cell cords of hepatocytes are separated by sinusoidal capillaries (Figure 1).

When we analyzed the postnatal pancreatic tissues, the histological differences between endocrine and exocrine pancreas were observed from the 5th day. Pancreatic lobulation and separation of endocrine units were clearly seen on the $15^{\text {th }}$ day (Figure 2,C,D). The exocrine and endocrine components of pancreas were histologically distincted. Within the pancreatic lobules, the exocrine pancreas was composed of closely arranged acini and the endocrine unit was consists of Langerhans islets scattered throughout the exocrine pancreas. 


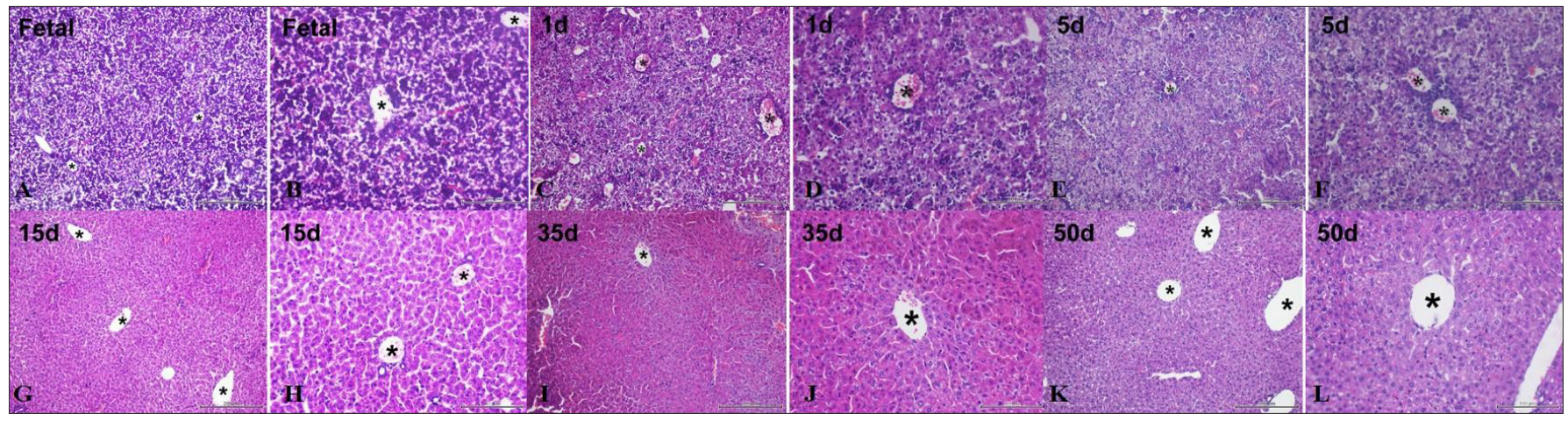

Figure 1. Histological examination of fetal and postnatal mouse liver.

Mouse fetal liver $(\mathrm{A}, \mathrm{B})$ and at 1 day $(\mathrm{C}, \mathrm{D}), 5$ days $(\mathrm{E}, \mathrm{F}), 15$ days $(\mathrm{G}, \mathrm{H}), 35$ days $(\mathrm{I}, \mathrm{J}), 50$ days $(\mathrm{K}, \mathrm{L})$ after birth. (Hematoxylin and eosin staining, stars: Central vein; the represantive pictures of A, C, E G, I, K: 20X and B, D, F, H, J and L: 40X magification, respectively)

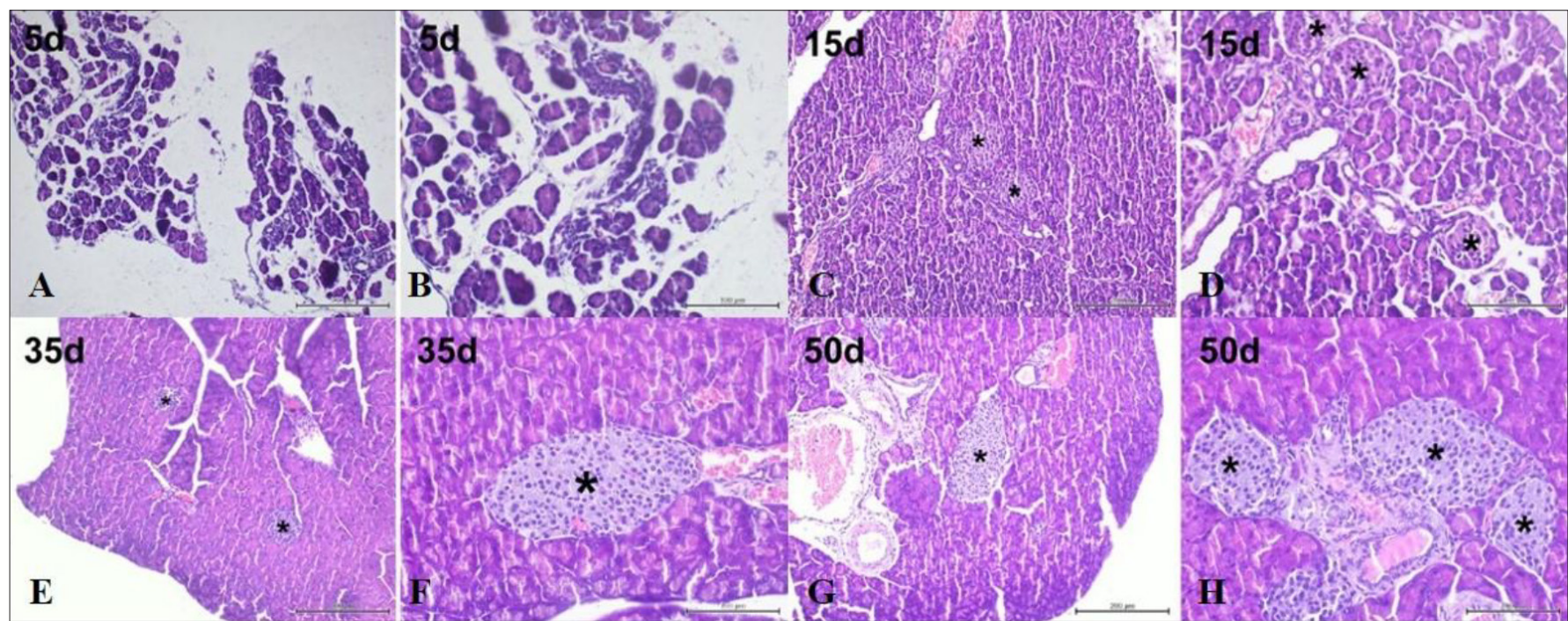

Figure 2. Histological examination of postnatal mouse pancreas

Mouse pancreas at 5 days $(A, B)$, at 15 days $(C, D), 35$ days $(E, F)$ and 50 days $(\mathrm{G}, \mathrm{H})$ after birth.Pancreatic lobulation were clearly seen on 15 th day .Exocrine pancreas was observed with brightly eosinophilic cytoplasm and slets of Langerhans (stars) are tightly-packed clusters of polygonal cells with pale eosinophilic cytoplasm. (Hematoxylin and eosin staining, stars: Endocrine pancreas; the represantive pictures of A,C,E and G: 20X; B,D,F and H: 40X magification, respectively.)

\section{Immunohistochemical Results}

We observed cytoplasmic and nuclear expression of p97/ VCP in hepatocytes of development liver. On day 1, p97/ VCP expression was seen in hepatocytes. On postnatal day 5, p97/VCP expression was increased compared to day 1 . At $15^{\text {th }}$ day, p97/VCP expression was increased compared to fetal and postnatal $1^{\text {st }}$ and $5^{\text {th }}$ days. Nuclear expression of P97/VCP was interesting seen on day 35. The immunostaining of $\mathrm{p} 97 / \mathrm{VCP}$ was weak around the central vein while it was strong at the periphery of the liver lobule on day 50 (Figure 3).

We showed that $\mathrm{p} 97 / \mathrm{VCP}$ immunexpression in postnatal pancreatic tissues, particularly in Langerhans islets from day 5. A polar expression of P97/VCP in Langerhans islet cells was observed on day 35. The most strong immunexpression of $\mathrm{p} 97 / \mathrm{VCP}$ was detected on the 50th day of the adult pancreas compared to other postnatal days (Figure 4).

Furthermore we investigated the immunoexpression of insulin and glucagon to confirm the langerhans islet cells in pancreas. In parallel with all these postnatal days,

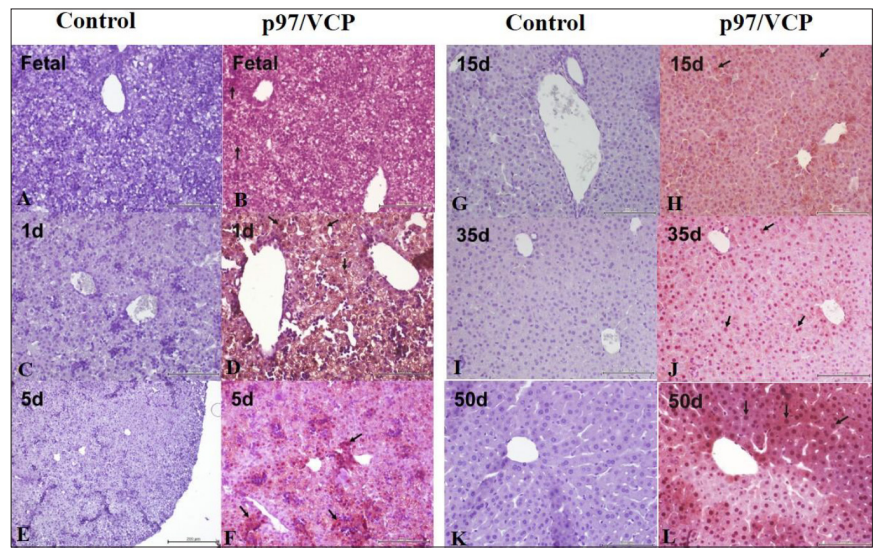

Figure 3. Immunostaining of P97 / VCP in fetal and postnatal mouse liver.

Immunohistochemical distribution of $\mathrm{p} 97 / \mathrm{VCP}$ in the mouse fetal liver $(\mathrm{A}, \mathrm{B})$ and at 1 day $(\mathrm{C}, \mathrm{D}), 5$ days $(\mathrm{E}, \mathrm{F}), 15$ days $(\mathrm{G}, \mathrm{H}), 35$ days $(\mathrm{I})),$,50 days $(\mathrm{K}, \mathrm{L})$ after birth. No significant staining was observed in the control. p97 / VCP expression was seen in hepatocytes (arrows) on day 1. On postnatal day 5, p97 / VCP expression was increased. Nuclear expression of P97 / VCP (arrows) was seen on day 35. The Immunostaining of p97 / VCP was weak around the central vein while it was strong at the periphery of the liver lobule on day 50.

positive controls of immune staining were confirmed by expressions of insulin and glucagon proteins in pancreas at 35 day (Figure 5,B,C). 


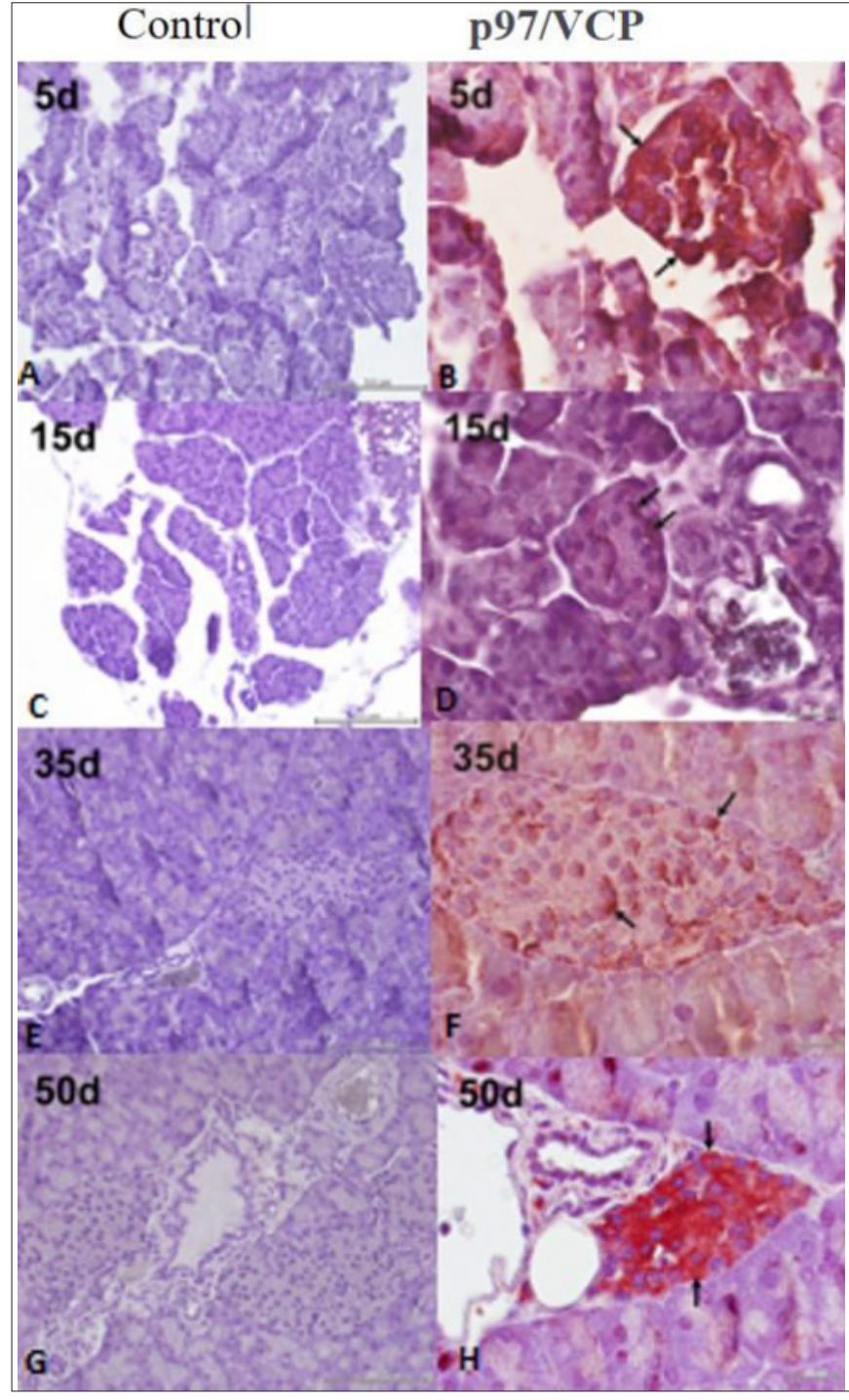

Figure 4. Imunostaining of P97 / VCP in postnatal mouse pancreas. Cellular localization of $\mathrm{p} 97 / \mathrm{VCP}$ in the postnatal mouse pancreas at 5 days $(\mathrm{A}, \mathrm{B})$ and at 15 days $(C, D), 35$ days $(E, F)$ and 50 days $(G, H)$ after birth. No significant staining was observed in the negative control. The immunoexpression of p97 / VCP was clearly observed in Langerhans islets (arrows) from day 5. The immunoexpression of $\mathrm{p} 97$ / VCP was weak at day 15 and the most strong expression (arrows) was observed in Langerhans islets at day 50th . (40X magnification).

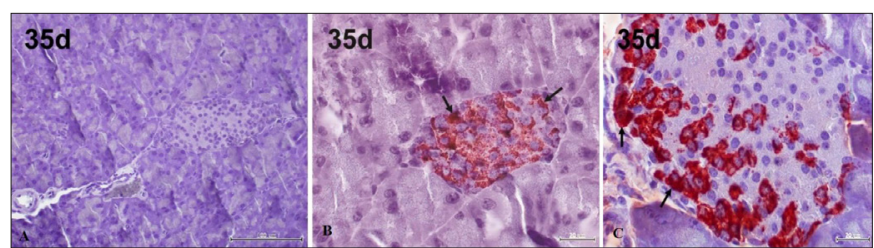

Figure 5. Immunostaining of insulin and glucagon in postnatal mouse pancreatic tissue.

A: Control, 35 days; B: Arrows indicate insulin positive cells, 35 days C: Glucagon positive cells (arrows) located at the periphery of Langerhans island, 35 days. ( $40 \mathrm{X}$ magnification).

H-SCORE analysis revealed that p97/VCP immunoreactivity increased from fetal to postnal liver and reached the highest expression level at day 50 (Figure 6,A). Additionaly, p97/VCP immunoexpression level detected in pancreas on day 5 . However, a slight decreased was observed in the pancreas of mice at 15 days and the highest expression level was at day 50 (Figure 6, B).

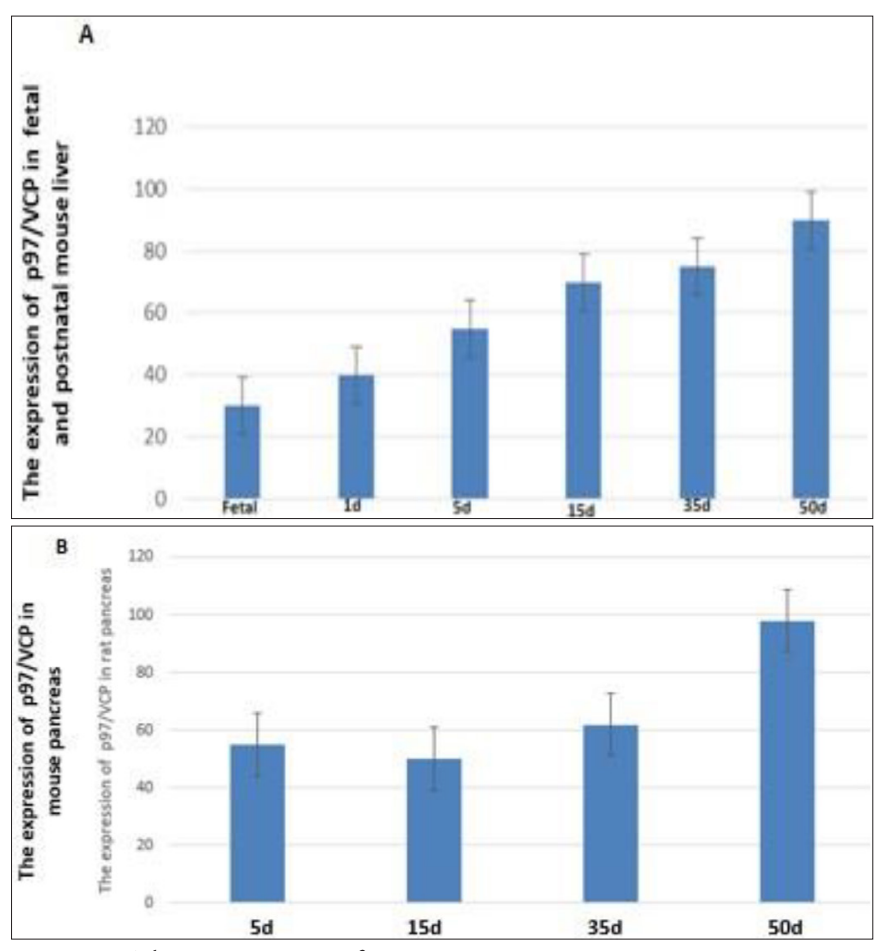

Figure 6. The H-SCOREs of $\mathrm{p} 97 / \mathrm{VCP}$ immunostaining intensities in the developing mouse liver (A) and pancreas (B). The data are represented as mean \pm SEM.

$\mathrm{H}$-SCORE analysis revealed that $\mathrm{p} 97 / \mathrm{VCP}$ immunoreactivity increased from fetal to postnatal mouse liver and reached the highest expression level at day 50 (A). p97/VCP immunoreactivity level detected on day5 and the highest expression level was at day 50 in mouse pancreas (B).

\section{DISCUSSION}

In the present study we investigated fetal and postanatal pancreas and liver histologically. We observed that normal histologic structure of mouse liver maintained in postnatal period and also liver lobulation was increased on day $15^{\text {th }}$. The histological differences between endocrine and exocrine pancreas were seen from day 5 . Pancreatic lobulation and separation of endocrine units were clearly seen on the $15^{\text {th }}$ day.

p97/VCP, a member of the AAA family, has a role as a chaperone and involve in the assembly, disassembly, and functional operation of protein complexes. Studies in the literature have showen that p97/VCP localized in the sperm head, midpiece and tail region. In the same study it has also reported that p97/VCP was negatively correlated with sperm concentration, motility and morphology. These results showing the different p97/VCP expression in fertile and infertile men and negative correlation between $\mathrm{p} 97 / \mathrm{VCP}$ expression and semen parameters suggest that $\mathrm{p} 97 / \mathrm{VCP}$ may be used one of the important factors for the evaluation of male infertility (16).

The UPS has important roles in many cellular processes via proteasomal degradation of ubiquitinated proteins. Recently published data has shown that Jab1/CSN5 interacts with p97/VCP and controls the ubiquitination 
status of proteins bound to $\mathrm{p} 97 / \mathrm{VCP}$ in mouse and human cells. This study indicate that p97/VCP expression overlapped with Jab1/CSN5 expression in gonocytes, spermatogonia, spermatocytes, Sertoli cells, spermatids and epididymal epithelialcells in the 5-, 15-, 30-and 60-day-old rat testis and epididymis (17).

Smad 1 is one of the signal transducers of BMP signaling and binds to several proteins involved in UPS. p97/VCP is required for the degradation of some UPS substrates. Recently published data show the cellular localization of Smads (Smad1 and pSmad1), the UPS proteins (p97/VCP, ubiquitin, Jab1/CSN5) and the interaction of proteins in the postnatal rat testis and epididymis. In 5-day-old rat testis, Smad1, phospho-Smad1, and p97/VCP were mainly expressed in gonocytes. In 15and 60-day-old rat testis, proteins were overlapped in spermatogonia, Sertolicells, and spermatocytes. The interaction between some of Smad proteins (Smad1 and phospho-Smad1) and UPS proteins (p97/VCP, JAb1/CSN5, ubiquitin) in the postnatal rat testis and epididymis suggests that UPS may play important roles in mediating BMP signaling during spermatogenesis (16).

Another study in the literature has shown the distribution and the colocalization of ubiquitin and p97/VCP in the developing rat retina.It has reported that the expression of ubiquitin significantly increased from 4-week-old to 72-week-old rats, however, p97/ VCP expression significantly decreased from 10-weekold to 72 -week-old rats in the retina. In the same study it has indicated that p97/VCP immunoreactivity in the retina significantly decreases after rats reach 10 weeks of age, whereas ubiquitin immunoreactivity increases with aging. These results suggest that an altered expression pattern of p97/ VCP and ubiquitin in the developing rat retina may assosciate with age-related retinal degeneration (18).

p97/VCP involve in cellular homeostasis by regulating endoplasmic reticulum-associated degradation (ERAD), mitochondrial-associated degradation (MAD), chromatin-associated degradation, autophagy, and endosomal trafficking. Researchers highlight the p97/ VCP as a therapeutic approach in neurodegeneration and cancer (19).

p97/VCP has enzyme functions related with protein homeostasis and quality control. Disruption of its normal function might be associated with the development of Parkinson's disease (PD). They have suggest that suggest that a decrease in the relative levels of VCP mRNA might serve as a biomarker for the development of pathology at the early clinical and preclinical stages of human PD (20).
The roles of p97/VCP in the cardiovascular system has been recently investigated.It has shown thar p97/VCP deficiency affects myocardial fibers and induces heart failure, while overexpression of $\mathrm{VCP} / \mathrm{p} 97$ eliminates ischemia/reperfusion injury and relieves pathological cardiac hypertrophy caused by cardiac pressure overload. VCP/p97 may be involved in the development of cardiovascular disease, and is anticipated to be a new therapeutic target (21).

Studies in the literature has reported that $\mathrm{VCP} / \mathrm{p} 97 /$ $\mathrm{Cdc} 48$ expression was positively correlated with cancer prognosis. Several studies have shown that $\mathrm{VCP} / \mathrm{p} 97 /$ Cdc48 might be a potential target in cancer therapy (22)

UPS and autophagy involve in protein quality control by degradation and clearance of damaged proteins. Several proteins in these pathways such as p97/VCP, Ubiquitin (Ub), Jab1/CSN5, p62, LC3B and Beclin 1 are essential in cancer. Recently,the researchers investigated the expression of UPS (p97/VCP, Ubiquitin, Jab1/CSN5) and autophagic (p62, LC3B, Beclin 1) proteins in human testicular tumors and cancer adjacent normal testicular tissues. The expression of p97/VCP, Ub, Jab1/CSN5, p62, LC3B and Beclin 1 was shown in different type of human testicular tumors. The results of the study have displayed elevated level of p97/VCP, Ub and Jab1/CSN5 expressions in contrast to the diminished expression of p62, LC3B and Beclin 1 in human testicular tumors, supporting a correlation between $\mathrm{p} 97 / \mathrm{VCP}$ and autophagic markers in testicular tumors (23).

In our study we investigated the immunoexpression of p97/VCP in fetal, postnatal and adoult liver and pancreas by using histological and immunocytochemical methods. We observed cytoplasmic and nuclear expression of P97/VCP in hepatocytes of development liver. The immunostaining of p97/VCP was seen in hepatocytes on day 1. Nuclear expression of p97/VCP was strongly observed on day 35 . When we analyzed the immunexpression of $\mathrm{p} 97 / \mathrm{VCP}$ in postnatal pancreas we determined the p97/VCP expression especially in Langerhans islets from day 5 . The most strong immunoexpression of p97/VCP was detected on the $50^{\text {th }}$ day of the adult pancreas when compared to postnatal days.

\section{CONCLUSION}

Our results showed the cellular localization of p97/ VCP in the fetal, postnatal and adult liver and pancreas and its expression was increased with the development. This study forms the basis for clinical studies in order to determine the function of $\mathrm{p} 97 / \mathrm{VCP}$ in liver and pancreatic cells. 


\section{ETHICAL DECLARATIONS}

Ethics Committee Approval: Kobay Animal Research Laboratory Local Ethics Committee (Date: 23.03.18, Decision No: 277).

Referee Evaluation Process: Externally peer-reviewed.

Conflict of Interest Statement: The authors have no conflicts of interest to declare.

Financial Disclosure: The authors declared that this study had received no financial support.

Author Contributions: All of the authors declare that they have all participated in the design, execution, and analysis of the paper and approved the final version.

Note: Oral presentation presented at 2. Ankara Yildırım Beyazıt Üniversitesi Tip Öğrenci Kongresi, Ankara, 19 21 September 2018.

\section{REFERENCES}

1. Ye Y, Meyer HH, Rapoport TA. The AAA ATPase Cdc48/p97 and its partners transport proteins from the ER into the cytosol. Nature 2001; 414: 652-6.

2. Xue L, Blythe E, Freiberger E, Mamrosh E, Hebert A, Reitsma J. Valosin-containing protein (VCP)-Adaptor Interactions are Exceptionally Dynamic and Subject to Differential Modulation by a VCP Inhibitor. Mol Cell Proteomics 2016; 15: 2970-86.

3. Ciechanover A. Intracellular protein degradation from a vague idea through the lysosome and the ubiquitin-proteasome system and on to human diseases and drug targeting: Nobel Lecture, December 8, 2004. Ann N Y Acad Sci 2007; 1116: 1-28.

4. Lord JM, Roberts LM, Stirling CJ. Quality control: another player joins the ERAD cast. Curr Biol 2005; 15: R963-R964.

5. Cao K, Nakajima R, Meyer HH, Zheng Y. The AAA-ATPase Cdc48/p97 regulates spindle disassembly at the end of mitosis. Cell 2003; 115: 355-67.

6. Voges D, Zwick P, Baumeister W. The $26 \mathrm{~S}$ proteasome: a molecular machine designed for controlled proteolysis. Annu Rev Biochem 1999; 68: 1015-68.

7. Song C, Wang O, Li H. ATPase Activity of p97-Valosin-containing Protein (VCP). J Biol Chem 2002; 278: 3648-55.

8. Maurizi M, Li C. AAA proteins: in search of a common molecular basis. EMBO Rep 2001; 2: 980-5.

9. Wang Y, Shirogane T, Liu D, Harper W, Elledge L. Exit from exit resetting the cell cycle through Amn1 inhibition of $\mathrm{G}$ protein signaling. Cell 2003; 112: 697-709.

10. Kondo H, Rabouille C, Newman R, et al. p47 is a cofactor for p97mediated membrane fusion. Nature 1997; 388: 75-8.

11.Dai RM, Li CC. Valosin-containing protein is a multi-ubiquitin chaintargeting factor required in ubiquitin-proteasome degradation. Nat Cell Biol 2001; 3: 740-4.

12. Meusser B, Hirsch C, Jarosch E, Sommer T. ERAD: the long road to destruction. Nat Cell Biol 2005; 7: 766-72.

13. Klein JB, Barati MT, Wu R, et al. Akt-mediated valosin-containing protein 97 phosphorylation regulates its association with ubiquitinated proteins. J Biol Chem 2005; 280: 31870-81.

14. Cayli S, Klug J, Chapiro J, et al. The COP9 signalosome interacts ATP-dependently with p97/VCP and controls the ubiquitination status of proteins bound to p97/VCP. J Biol Chem 2009; 284: 34944-953.

15. Ozsoy A, Cayli S, Sahin C, Ocakli S, Sanci T, İlhan D. Altered expression of $\mathrm{p} 97 / \mathrm{Val}$ osin containing protein and impaired autophagy in preeclamptic human placenta. Placenta 2018; 67: 45-53

16. Cayli S, Erdemir F, Ocaklı S. Interaction between smad1 and p97/ vcp in rat testis and epididymis during the postnatal development. Reprod Sci 2012; 19: 190-201.

17. Cayli S, Ocakli S, Erdemi F, et al. Developmental expression of p97/VCP (Valosincontaining protein) and Jab1/CSN5 in the rat testis and epididymis. Reprod Biol Endocrinol 2011; 9: 117.

18. Ortak H, Cayli S, Tas U, Ocakli S, Söğüt E, Demir HD. Expression of p97/VCP and ubiquitin during postnatal development of the degenerating rat retina. J Mol Hist 2012; 43: 17-25.

19. Donna H, David K, Peter W. p97: An emerging target for cancer, neurodegenerative diseases, and viral infections. J Med Chem 2020; 12: 1892-907.

20. Anelya A, Margarita R, Elena F, Alexey K, Olga D, Kseniya D. VCP expression decrease as a biomarker of preclinical and early clinical stages of Parkinson's disease. Sci Rep 2020; 10: 827.

21.Hongyang S, Yizhong P, Weijian H, Ning Z, Dao W. Emerging role of $\mathrm{VCP} / \mathrm{p} 97$ in cardiovascular diseases: novel insights and therapeutic opportunities. Biochem Soc Trans 2021; 49: 485-94.

22.Lan B , Chai S, Wang P, Wang K. VCP/p97/Cdc48, A linking of protein homeostasis and cancer therapy. Curr Mol Med 2017; 17: 608-18.

23. Nakkas H, Gumuskaya Ocal B, Kipel S, et al. Ubiquitin proteasome system and autophagy associated proteins in human testicular tumors. Tissue Cell 2021; 71: 101513. 